\title{
A TECHNOLOGY TO (RE-)CONNECT OPTICAL FIBRES EMBEDDED IN COMPOSITE STRUCTURES
}

\author{
EMUS 2020 \\ J. WINDELS ${ }^{1}$, J. MISSINNE ${ }^{2}$, E. VOET ${ }^{1}$, G. VAN STEENBERGE ${ }^{2}$, G. LUYCKX ${ }^{1}$ \\ Com\&Sens BV \\ Begoniastraat 17 PB2 B-9810 Eke Belgium \\ gbeauduin@com-sens.euwww.com-sens.eu
}

Keywords: Fiber Bragg Gratings, Optic Fibers, Structural Health Monitoring

\begin{abstract}
Optical fibre Bragg grating (FBG) sensors are commonly used for structural health monitoring in composite materials, since their small dimensions, high sensitivity and multiplexing capacity are a good match with the structures to be monitored. We present a method for making a low-loss connection to an FBG optical fibre sensor that is integrated in a fibre reinforced composite structure. The method allows to both manufacture composite structure without taking special precautions to prevent damage to the connecting fibre and (if needed) to repair the connecting fibre, while optimizing the optical connection efficiency even if both fibres have dissimilar mode field diameters.
\end{abstract}

\section{INTRODUCTION}

Optical Fibre Bragg grating sensor technology (FBG) is the most promising technology to implement self-sensing features in composite materials. By integrating these sensors inside the composite material (as opposed to sensors that are placed on the surface), they allows insitu measuring of material deformations and monitoring of the integrity of the structure. At the same time, integrating the sensors inside the composite material will protect the FBG from the potentially harsh environment. However, despite these clear advantages, the industry adoption of these FBG sensors in the production and monitoring of composite structures has been lagging due to a practical concern: the location where the fibre enters the composite material, the so called 'in- or egress' point, is fragile, and complicates the production process of the composite structure. Typically, once the optical fibre is fractured at the egress point, the connection can not be repaired and the functionality of the embedded FBG sensors is lost. Attempts to make the fibre more robust at the egress point, such as adding tubing or incorporating a discrete optical connector at the composite edge all have their drawbacks[1,2,3]. Typically, they require adaptations to the mould to lead out the fibre or protect the connector, do not allow for trimming of excess material after the composite production and tend to have an impact on the mechanical properties of the resulting composite structure. To tackle these challenges, we present a low- 
loss optical connection method that has no impact on the composite production process or mould design, and can be performed after the composite production is complete (including trimming if desired).

\subsection{Fibre Bragg grating sensors}

The fibre optic sensors applied in this paper are embedded fibre Bragg gratings (FBGs) used for strain measurements in the composite laminate. An FBG is a periodic change of the refractive index of an optical fibre over a certain length (typically 1 to $10 \mathrm{~mm}$ ). In one single fibre, a large number of sensor points can be multiplexed when multiple FBGs are present in the fibre at different positions. The measuring principle is shown in

Figure 1. When light containing multiple wavelengths is coupled into the optical fibre, only one wavelength of the light is reflected (depending on the effective refractive index and the period of the grating, $\Lambda$, ). When the FBG is elongated the reflected wavelength increases, vice versa when the FBG is compressed the wavelength decreases (a one on one relation exists with the deformation of the FBG). The sensors used in this work are Draw Tower Gratings (DTG $\left.{ }^{\circledR},[4]\right)$.

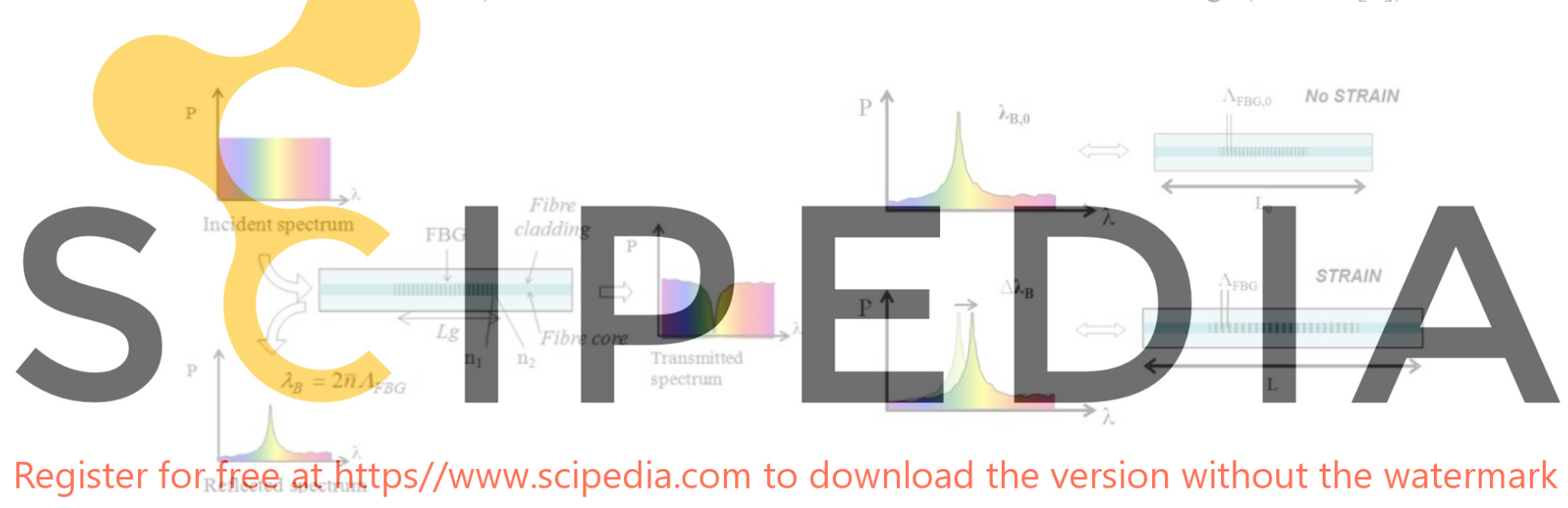

Figure 1 left principle of an $\mathbb{F B G}$ sensor, right refiracted wavelength of the $\mathbb{F B G}$ in unstrained and strained condition (FBGS INT.).

\section{PROBLEM DEFINITION AND STATE OF THE ART}

\subsection{State of the art}

Optical fibre sensors embedded in composite materials are of great interest for measuring insitu material deformations of composite structures. However, because the current fibre optic connection solutions have drawbacks with respect to ease of application, time occupation, installation cost, repairability, and compatibility with the composite production process (distortion), there is still a lack of industry uptake of this technology. The most relevant existing solutions are illustrated in Figure 1 and described below. 


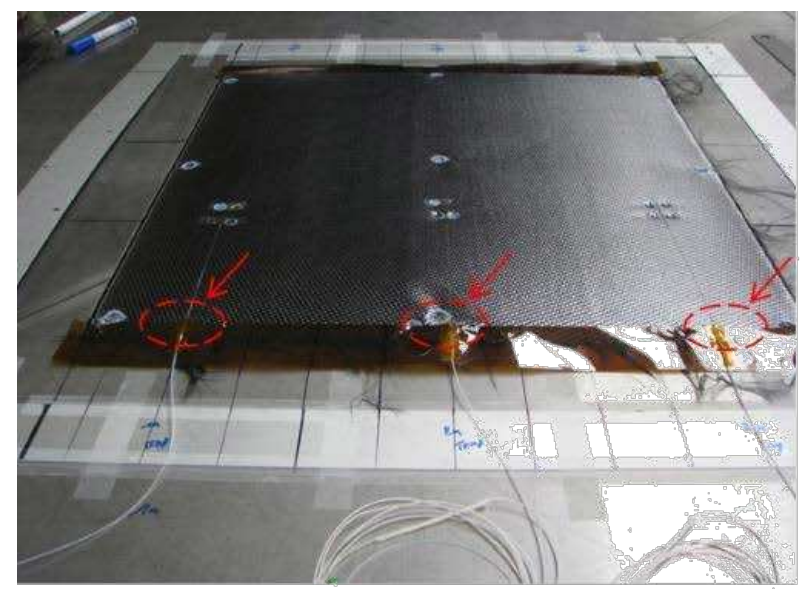

1. Protecting fibre egress points during fabrication
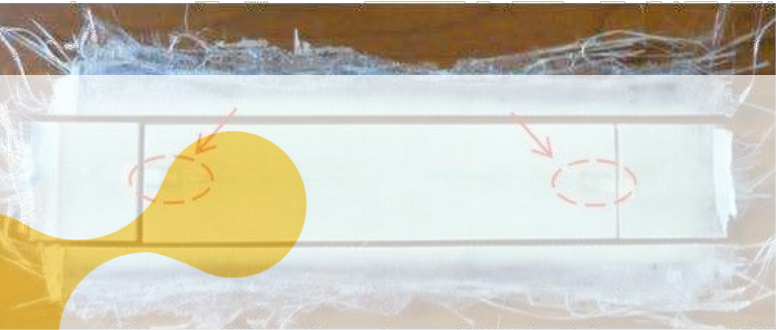

3. embedding a miniature teflon end-piece
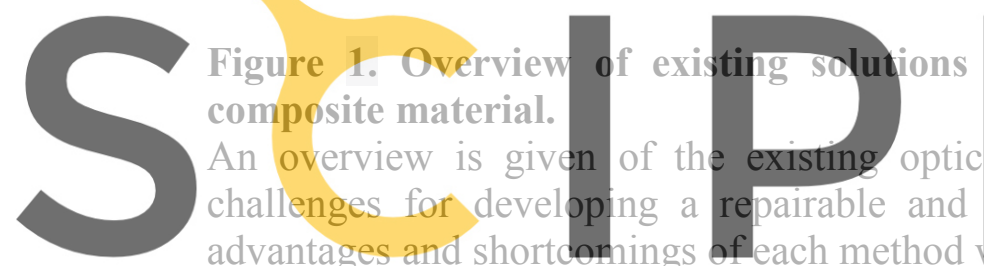

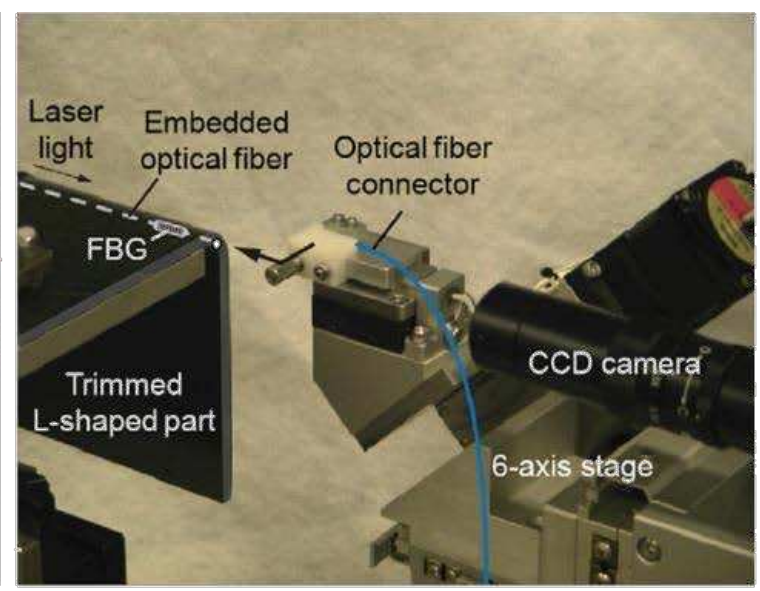

2. Glueing the optical fibre

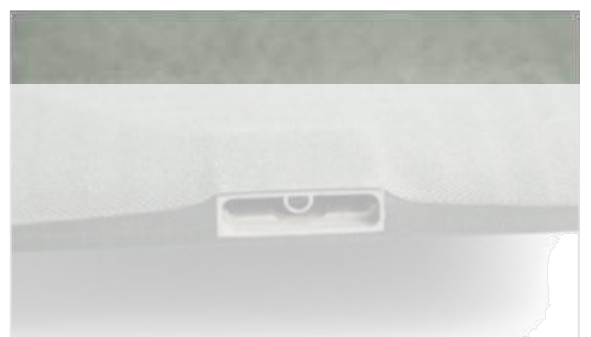

4. Discrete connector on the composite edge
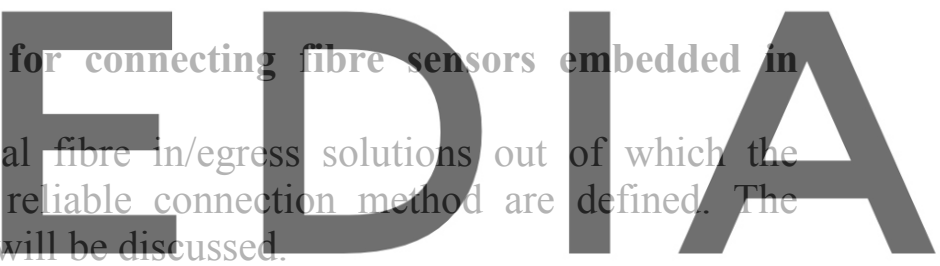

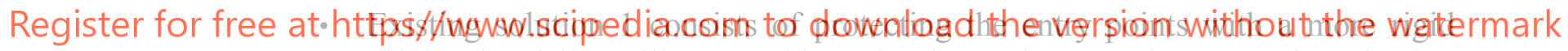
material (tube, foil or silicone). This option is cumbersome in terms of production and not all orientations or positions of the embedded fibre are possible. In addition, when using closed mould composite technologies, an adaptation of the mould is needed. Furthermore, it is hardly possible to trim edges.

- Existing solution $2^{1}$ consists of glueing the optical fibre to the composite. An index-matching glue is used to make the connection. Although this method allows for edge trimming of composite and is compatible with the composite production process, the downsides are high fibre coupling losses (in the order of $\mathrm{dBs}$ ) and the technique is restricted to connecting a fibre normal to the edge of the composite part.

- Existing solution $3^{2}$ consists of embedding a miniature Teflon end-piece in the composite [4]. This end-piece should exactly be placed in the composite at the position of the edge trimming (small tolerances). The method only allows entry points at the sides of the composite, and although miniature, still distorts the composite at its edges. A previous, bigger version of this technique has already been presented in 2000, see Sjogren, SMS $2000^{3}$. 
- Existing solution 4 consists of placing discrete connectors on the side or on top of the composite laminate. An overview of these techniques is shown in Green_SMS_1999 ${ }^{4}$. There are some recent connectors developed by Deutsch connector ${ }^{5}$. Because of the size of the connectors, there always is undesired distortion of the composite and the method clearly involves extra production steps during the application of the connector (extra reinforcement layers, adaption of moulds,...). Furthermore, only limited edge trimming is allowed not to damage the connectors.

\section{Main challenges of the connection of an embedded fibre sensor and novelty content of the current concept:}

- challenge \#1: When fabricating composite laminates, pieces or real structures, often the edges of that structure are trimmed prior to use. By trimming the edges, all embedded fibres will normally be cut as well and left without connector. Novelty content: With the method proposed in this document, (re-) connecting of these embedded sensors is possible.

- challenge \#2: Ingress/egress techniques existing are mainly based on feed through designs and external fibre coupling. This may involve mould modifications which is not preferred. Novelty content: The method described here will be able to couple the fibre directly at the edge or surface of the composite and allows connecting or repairing of the connection at any time.

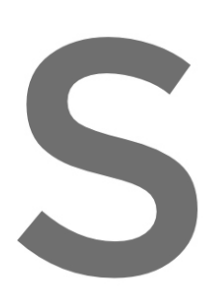

challenge \#3:
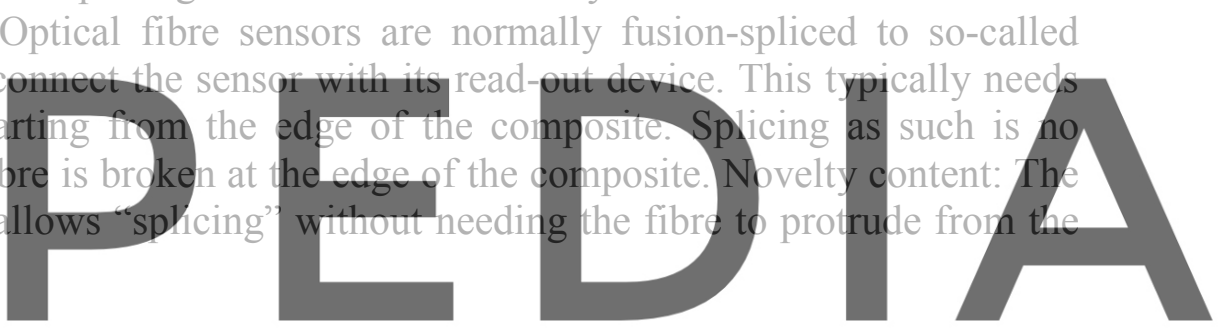

2.1 Composite edge-connect

Register for free at https//www.scipedia.com to download the version without the watermark

To tackle the above-mentioned challenges, a novel concept is described in this letter which is based on a low-loss optical 'splice', which is packaged to ensure mechanical robustness. The technique does not require changes to the composite production process: after production, the embedded fiber is exposed by making a cross-sectional cut, the external fiber is aligned with it and then a direct optical splice is mad.

During production of the composite structure, one or more FBG sensor fibres (DTG ${ }^{\circledR}$ technology, from FBGS International) are included at the desired location in the lay-up of the composite structure. The diameter of these DTG sensor fibres is $125 \mu \mathrm{m}$, and they are factorysupplied with an Ormocer ${ }^{\circledR}$ coating, leading to total diameter of $195 \mu \mathrm{m}$.

After production of the composite structure, the embedded FBG sensor is exposed by making a cross-sectional cut perpendicular to the sensor fibre. The surface is then locally polished using P2400 and P4000 abrasive papers. Then, an external readout fibre, which typically will be a lowcost standard single mode telecom fibre, is actively aligned with the embedded sensor fibre, and a direct 'optical splice' is made. Since the numerical aperture and mode field diameter of both types of fibres are different, coupling losses are present when these are directly coupled to each-other. The 'optical splice' is implemented using an intermediate self-written waveguide (SWW), which connects the 2 fibres. This SWW is formed by launching UV light through the readout fibre while the intermediate space between the sensor fibre and readout fibre is filled 
with an appropriate UV curable resin. Due to an increase in refractive index upon polymerization, which starts at the tip of the fibre where the intensity is the highest, a lens-like structure is formed at the end of the fibre tip. This lens-like structure then concentrates the UV light in front of the fibre tip, leading to a progressively growing polymerized region with increased refractive index in front of the fibre forming a waveguide structure.[5] After the formation of the SWW, the assembly is mechanically fixated by gluing a bracket around the edge of the composite, which also provides strain relief to the splice.

\section{EXPERIMENTATION AND RESULTS}

\subsection{Alignment}

As mentioned in the introduction, the sensor fibre and connection fibre are actively aligned with each-other using a precision optical stage. The alignment can be either fully manual or be semiautomated. In the case of automated alignment, there is a first coarse manual alignment to bring both fibres within the range of motion of a precision optical stage, which is typically only a few $\mathrm{mm}$. If a redundant connection to the integrated sensor fibre is available, which was ensured during the experimental work below, but is far from guaranteed in practical situations, this coarse alignment can be assisted by shining visible (e.g. red) light through the redundant connection and aligning the beam with the readout fibre. This can be easily accomplished
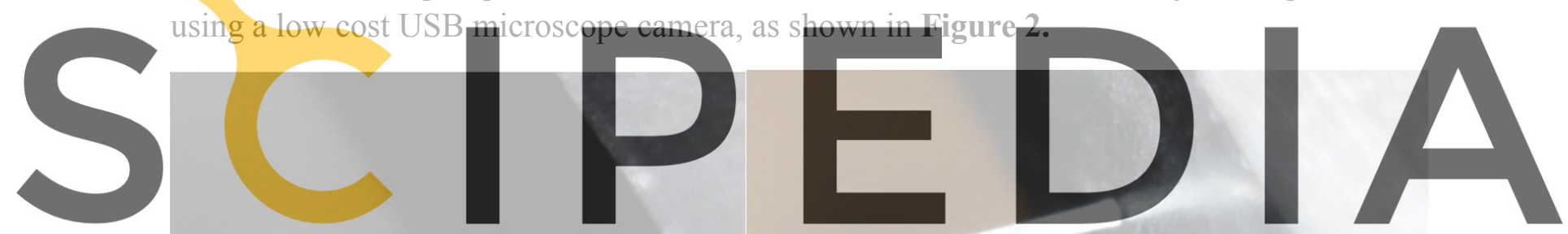

for free at https//www.scipedia.com to download the version without the watermark

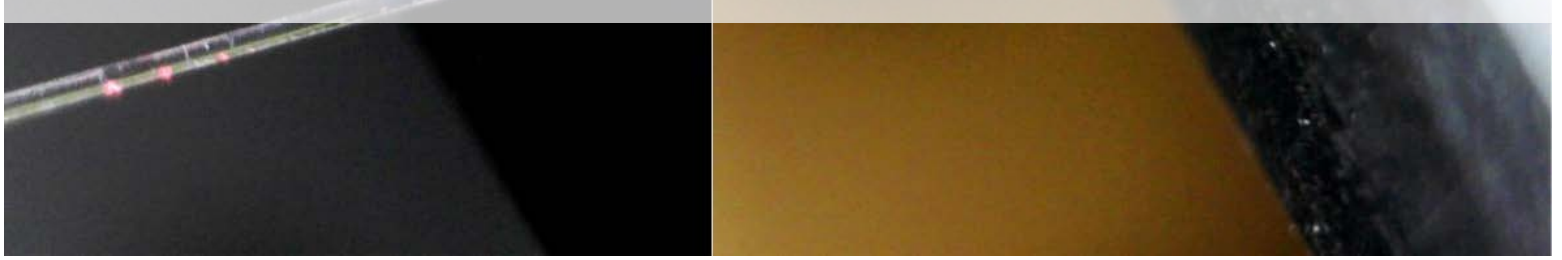

Figure 2 Coarse alignment of readout fibre to sensor fibre integrated in CFRP using visible light from redundant connection(left.) The readout fibre has been stripped and cleaved and has a diameter of $125 \mu \mathrm{m}$. When the visible laser is turned off, finding the location of the sensor fibre becomes challenging (right.)

However, when no redundant connection is available, it is fairly difficult to distinguish the $195 \mu \mathrm{m}$ diameter integrated DTG sensor fibre from the GFRP or CFRP using the USB microscope. This is mainly due to a practical consideration: the camera needs to be placed at an angle, since the readout fibre is blocking the camera from being perpendicular to the cross section. Fortunately, the presence of the readout fibre enables us to locate the embedded fibre. A first potential approach is by taking advantage of the difference in reflectivity between the embedded fibre and the surrounding GFRP or CFRP by measuring the reflected power. An 
alternative approach consists of detecting the Bragg peaks in the reflected spectrum. The first approach using the reflected power provides a relatively straightforward measure for the location of the fibre: while scanning an area for the presence of an embedded fibre, each location corresponds with a single value: a measure of the reflected optical power, which does not need to be calibrated and can be easily interpreted by software. This does require the presence of a way to measure the reflected power, such as a circulator combined with a power meter or a fibre coupled photodiode. A second approach is inspecting the reflected spectrum for the presence of Bragg reflection peaks, using an optical spectrum analyzer or a commercial fibre interrogator. This allows for aligning the core of the readout fibre with the core of the sensor fibre using the same hardware that will already be present to interrogate the sensors, but is not as straightforward as it may appear at first. During the alignment process, an air gap is present between the readout fibre and the sensor fibre, which creates a Fabry-Pérot cavity. Because of this, the typical Bragg spectrum peak(s) are superimposed on an interference pattern related to the wavelength and distance between the two end-facets, as shown in Figure 3. This somewhat complicates the automation of the alignment, although we are currently having good initial first results with a discrete wavelet-based Bragg peak detection algorithm.

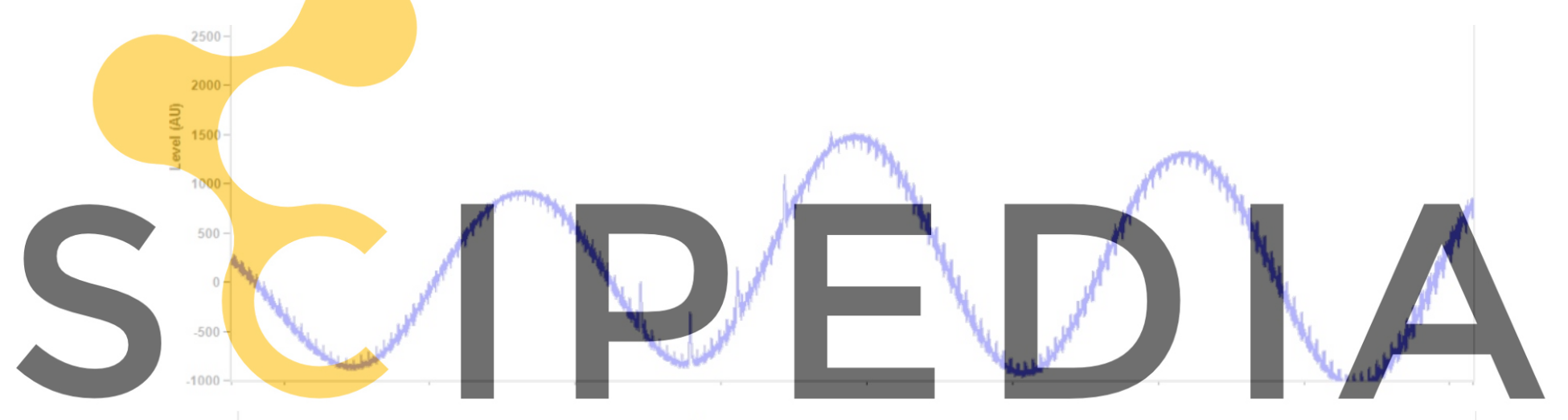

Register for free at https//www.scipedia.com to download the version without the watermark

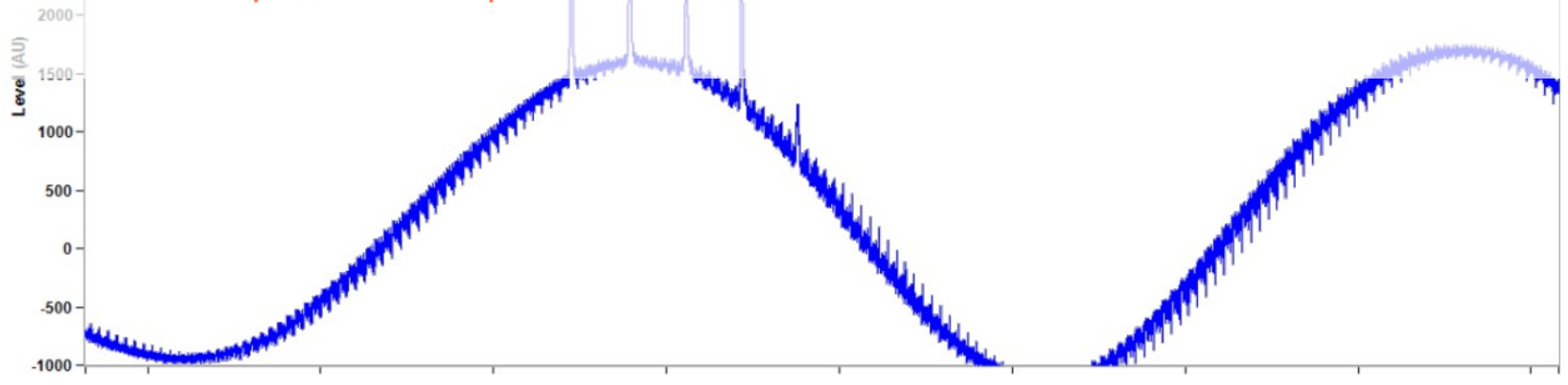

Figure 3 Five Bragg reflection peaks superimposed on Fabry-Perót reflection spectrum for perfectly aligned sensor and readout fibres, with $50 \mu \mathrm{m}$ separation(top) and in nearcontact (bottom)

An example of a reflection signature of an embedded DTG fibre in a composite structure is shown in Figure 4(left), while Figure 4(right)shows the signature of the wavelet-based Bragg 
peak reflection. Note the difference in scale: if the Bragg reflection peaks are used for alignment, only the location of the core is detected instead of the location of the entire fibre.
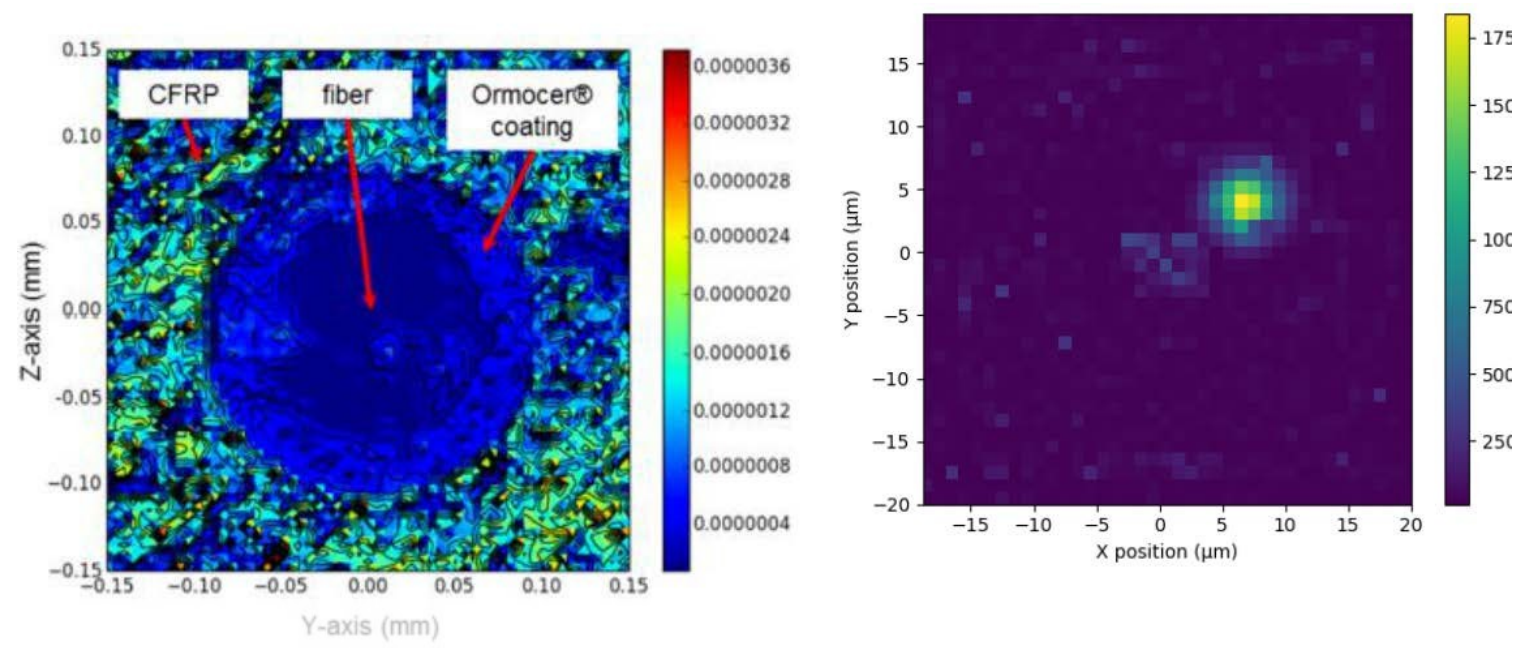

Figure 4 Signature of reflected power (arbitrary units) from scanning over an area containing a DTG fibre (left), signature of sensor fibre core location detection using wavelet based detection algorithm of the Bragg peaks(right). Units are arbitrary, and not comparable between the 2 methods.

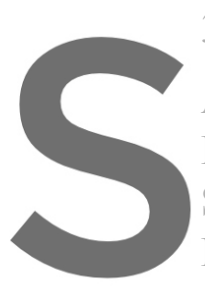

\subsection{Design and material choice}

A certain gap is maintained between both fibres which is Experimentally, $50 \mu \mathrm{m}$

SWW and providing a During optimization in lab conditions and to
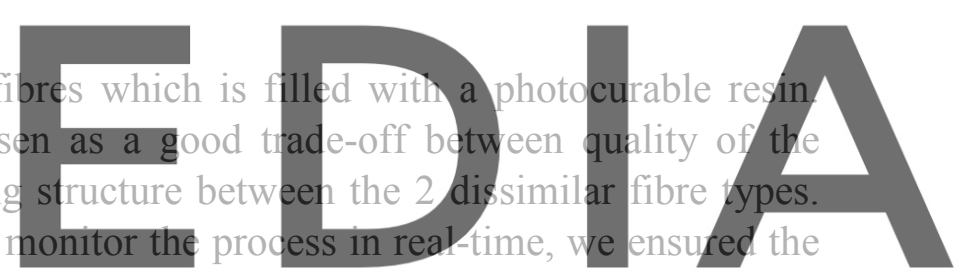

presence of a redundant connection to the embedded fibre. This allows for measuring the

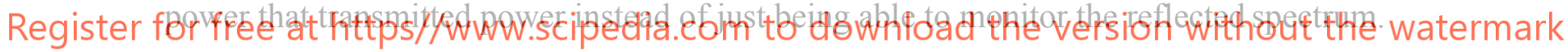

\subsection{Simulations}

Using Finite Difference Time Domain (FDTD) simulations with an empirical model of graded-index SWWs, we simulated the ideal SWW RI profile. The correct RI profile is crucial in achieving maximum transmission of the signal between the 2 dissimilar fibres. This is documented in a previous publication[6], and is illustrated in Figure 5. The simulated maximum efficiency corresponds with $0.42 \mathrm{~dB}(\mathrm{~T}=90.7 \%)$ loss for a $50 \mu \mathrm{m}$ long SWW, while direct butt-coupling of the 2 fibres leads to a loss of almost $1 \mathrm{~dB}$. In theory, it is possible to increase the transmission to $94.4 \%$ using a $90 \mu \mathrm{m}$ long SWW, but experimentally this lead to lower quality of the fabricated SWW structures. 


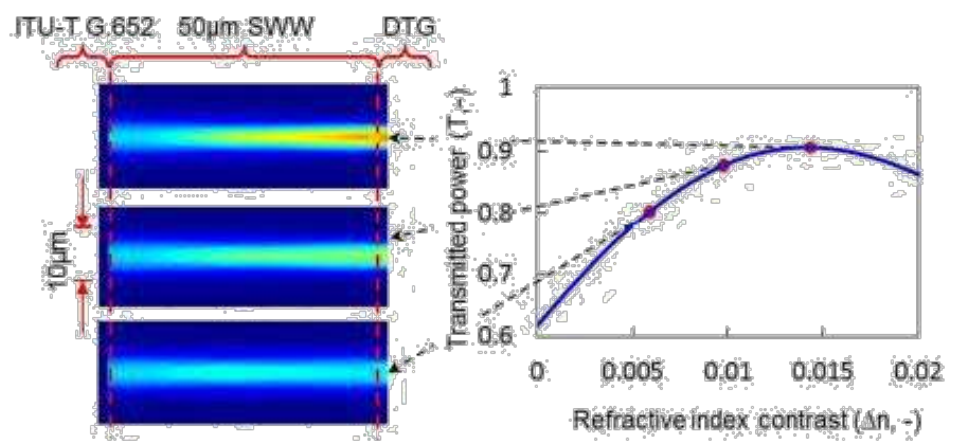

Figure 5 Simulated fraction of transmitted power between connection fibre-SWW (50 $\mu \mathrm{m}$ long)-DTG fibre connection as a function of the refractive index contrast in the SWW. The 2D color plots show the power distribution in a longitudinal cross-section

\subsection{Fabrication and measurements}

The fabrication parameters of the SWW (illumination power and time) were optimized by evaluating the transmitted power during fabrication (by ensuring the other side of the DTG fibre was accessible and connected to a power meter). Maximum transmission for a $50 \mu \mathrm{m}$ long SWW was achieved using a 30s illumination time using the fibre coupled laser diode at a power of 20 $\mu \mathrm{W}$ (measured at the tip of the connection fibre), while simultaneously performing a flood exposure from the outside at $5 \mathrm{~mW} / \mathrm{cm}^{2}$. To verify the formation of a SWW, the insertion loss was monitored as a function of the llumination time. The reference
fibres being perfectly aligned with mininal separation (near cont
of uncured photocurable resin. The initial $1.5 \mathrm{~dB}$ at t=0 im the gr
of 50 m between the 2 fibres, with the gap being filled with $p$ illumination is started,
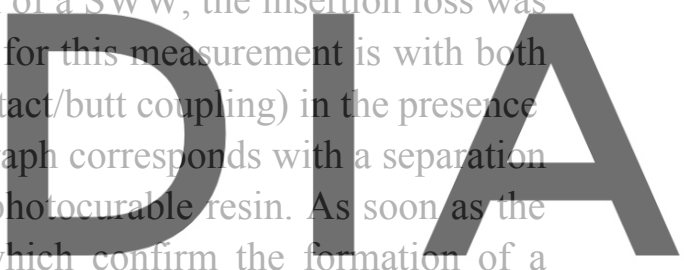

SWW. Because the energy density of the UV laser light that is coupled through the fibre is much

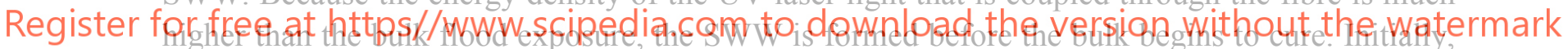

this SWW is surrounded by the still-liquid uncured cladding material and has a sufficiently high RI contrast, which allows for the transition between the 2 fibre types to work as it does in simulation. It can be seen that the insertion loss decreases to less than $0 \mathrm{~dB}$, demonstrating the improvement over direct butt-coupling. Unfortunately, in a later phase ( $\mathrm{t}>10 \mathrm{~s}$ ), the loss increases again, due to the cladding material starting to polymerize with the flood UV exposure, which reduces the RI contrast again. This is an inherent limitation of using the same polymer for core and cladding, as the maximum RI contrast is limited. It is possible to achieve higher contrast by using a 2-polymer approach, where the unexposed and uncured cladding material is removed in a development step and a second type of polymer is applied and cured as the final cladding. However, in practice this approach is not desirable due to the fragile nature of the SWW when the cladding is not present. Even if the minimum theoretical loss cannot be achieved in reality with the photocurable resin we used, the final insertion loss after $30 \mathrm{~s}$ illumination is still $0.5 \mathrm{~dB}$ lower than the case of direct butt-coupling (i.e. the reference value). Furthermore, the use of an intermediate SWW allows greater connection flexibility. For example, in case the embedded fibre is not cut perpendicularly, the free space region between its end-face and that of the external fibre can be bridged by the SWW. 


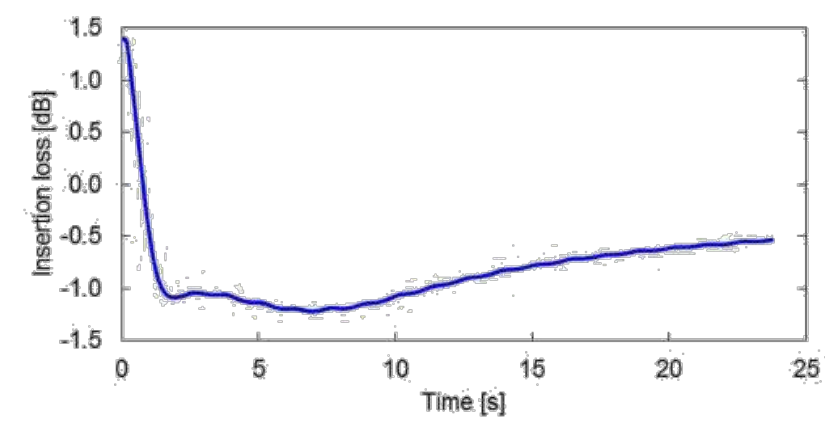

Fig 4. Insertion loss through an ITU-T G.652 - 50 um long SWW - DTG fiber connection during SWW fabrication compared to the reference (fibers at 0 min separation, with uncured NOA68 material in between):

\section{Figure 6 Measured insertion loss during fabrication of SWW}

To make the connection between the composite test-piece with the embedded FBG sensors mechanically stable and to provide strain relief, the SWW assembly is protected by a 3D printed bracket.
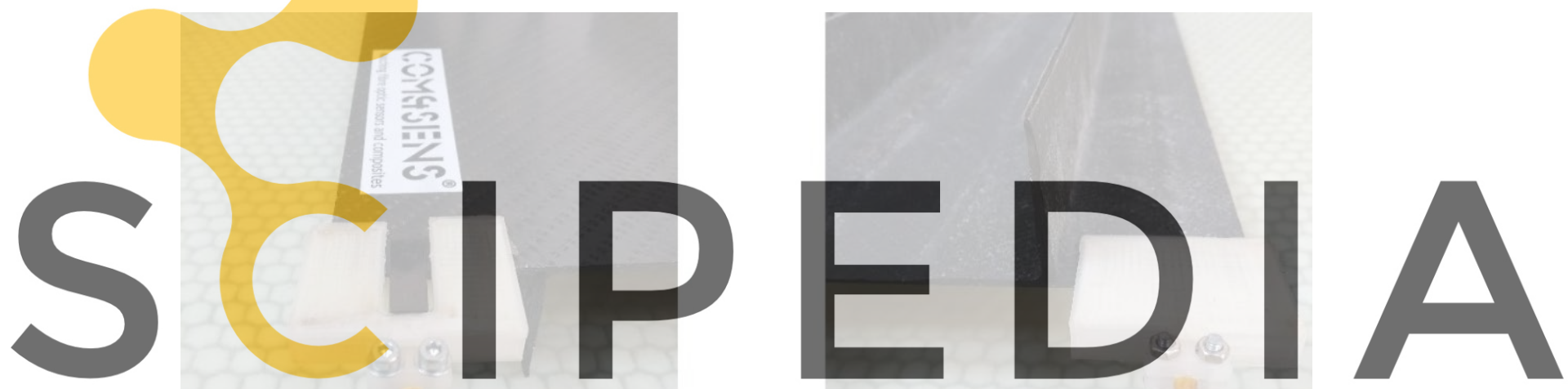

Register for free at https//www.scipedia.com to download the version without the watermark

Figure 7 Camera images of a 3d printed bracket to reinforce the SWW connection on a CFRP test-piece with embedded sensors

\section{CONCLUSIONS}

In this paper, we discussed a method that allows connecting an external read-out fibre to a sensor fibre that is embedded in a composite structure, without requiring any special precautions or modifications during the composite manufacturing process. By using an intermediate SWW between the read-out fibre and the sensor fibre, it is possible to optimize the coupling between these 2 dissimilar fibre types compared to direct butt-coupling. Despite not achieving the theoretically possible connection loss due to limitations of the SWW materials that were used in this work, we have demonstrated that it is possible to produce a high quality optical connection that allows for tracking the signal from the FBG sensors with a commercial interrogator. 


\section{ACKNOWLEDGEMENTS}

This work was supported by the European Space Agency (ESA), under contract nr. 4000114365/15/NL/CBi/GM.

This research was carried out within the project "QUALIFY - Enabling Qualification of Hybrid Joints for Lightweight and Safe Maritime Transport", co-funded by the INTERREG 2SeasMers Zeeën programme http://www.interreg2seas.eu/qualify

\section{interreg}

2Seas Mers Zeeën

\section{REFERENCES}

1. Kang, H. K., Park, J. W., Ryu, C. Y., Hong, C. S., \& Kim, C. G. (2000). Development of fibre optic ingress/egress methods for smart composite structures. Smart Materials and Structures, 9(2), 149.

2. Sjögren, A. (2000). Manufacturing technique for embedding detachable fiber-optic connections in aircraft composite components. Smart materials and structures, 9(6), 855.

3. Green, A. K., \& Shafir, E. (1999). Termination and connection methods for optical fibres embedded in aerospace composite components. Smart materials and structures, 8(2), 269.
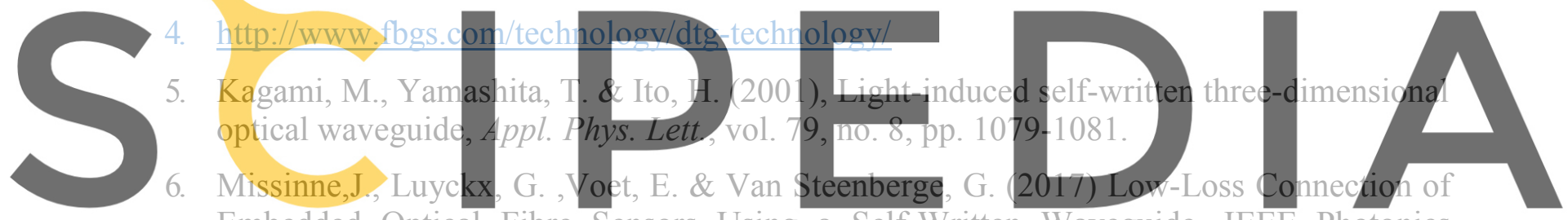

Embedded Optical Fibre Sensors Using a Self-Written Waveguide. IEEE Photonics

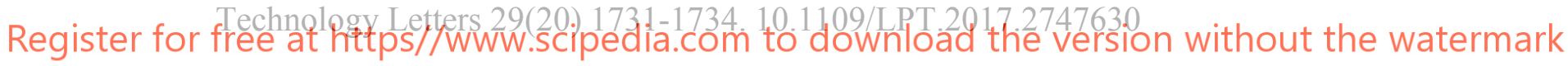

